\title{
DNA-Origami-Aided Lithography for Sub-10 Nanometer Pattern Printing ${ }^{\dagger}$
}

\author{
Isaac Gállego 1,**, Brendan Manning ${ }^{1}$, Joan Daniel Prades 2,*, Mònica Mir 3,4, \\ Josep Samitier ${ }^{2,3,4}$ and Ramon Eritja ${ }^{1,4, *}$ \\ 1 Institute for Advanced Chemistry of Catalonia (IQAC), Spanish National Research Council (CSIC), Spain; \\ bmanning@iqac.es \\ 2 Department of Engineering: Electronics, University of Barcelona, Barcelona, Spain; jsamitier@ibec.cat \\ 3 Nanobioengineering Laboratory, Institute for Bioengineering of Catalonia (IBEC), Barcelona, Spain; \\ mmir@ibec.cat \\ 4 Networking Center in Bioengineering, Biomaterials and Nanomedicine (CIBER-BBN), Barcelona, Spain \\ * Correspondence: igallego@mrc-lmb.cam.ac.uk (I.G.); dprades@el.ub.edu (J.D.P.); recgma@cid.csic.es (R.E.) \\ + Presented at the Eurosensors 2017 Conference, Paris, France, 3-6 September 2017.
}

Published: 8 August 2017

\begin{abstract}
We report the first DNA-based origami technique that can print addressable patterns on surfaces with sub-10 $\mathrm{nm}$ resolution. Specifically, we have used a two-dimensional DNA origami as a template (DNA origami stamp) to transfer DNA with pre-programmed patterns (DNA ink) on gold surfaces. The DNA ink is composed of thiol-modified staple strands incorporated at specific positions of the DNA origami stamp to create patterns upon thiol-gold bond formation on the surface (DNA ink). The DNA pattern formed is composed of unique oligonucleotide sequences, each of which is individually addressable. As a proof-of-concept, we created a linear pattern of oligonucleotide-modified gold nanoparticles complementary to the DNA ink pattern. We have developed an in silico model to identify key elements in the formation of our DNA origami-driven lithography and nanoparticle patterning as well as simulate more complex nanoparticle patterns on surfaces.
\end{abstract}

Keywords: DNA nanotechnology; lithography; nanopatterning; gold nanoparticles; metasurfaces

\section{Introduction}

The programmability [1] and self-assembly properties of DNA provides means of precise organization of matter at nanoscale. For the past decade, researchers have used a method named "DNA origami" [2] by which a long, circular single stranded DNA scaffold (the genome of the M13 virus) is folded to a specific shape using hundreds of short oligonucleotides (staple strands) that have been rationally designed. DNA origami allows the folding of DNA into two-dimensional and threedimensional nanostructures. This property has been utilized here to organize biomolecules, nanophotonic and electronic components with a resolution of $6 \mathrm{~nm} /$ pixel; surpassing the limitations of top-down methodologies [3].

Specifically, two-dimensional DNA origami has been placed on technologically relevant substrates and used as a platform to organize other chemical species [4]. However, to the best of our knowledge, DNA origami methodology has not been utilized to transfer and covalently bind the patterns on surfaces with sub-10 $\mathrm{nm}$ resolution. Herein we report on the use of a two-dimensional DNA origami as a template that bears pre-programmed patterns that can be lithographically transferred to a surface [5] (Figure 1). 


\section{The Method}

Our printing method (or stamping method) utilizes DNA origami "tall rectangle" containing modified staple strands in the programmed positions (DNA origami stamp) acting as DNA ink (Figure 1). After adsorption, the modified staples can then react with the surface printing the DNA pattern (Stamping step). Then, the lithographed DNA pattern is exposed after denaturation of the DNA origami stamp frame (Unmasking step). Once the DNA ink pattern is exposed, can be utilised to organise other chemical species over the surface via hybridisation with the printed DNA ink pattern (Development step, Figure 1 step 3).

$\begin{array}{lll}\text { 1) Stamping of DNA ink } & \text { 2) Unmasking } & \text { 3) Development }\end{array}$

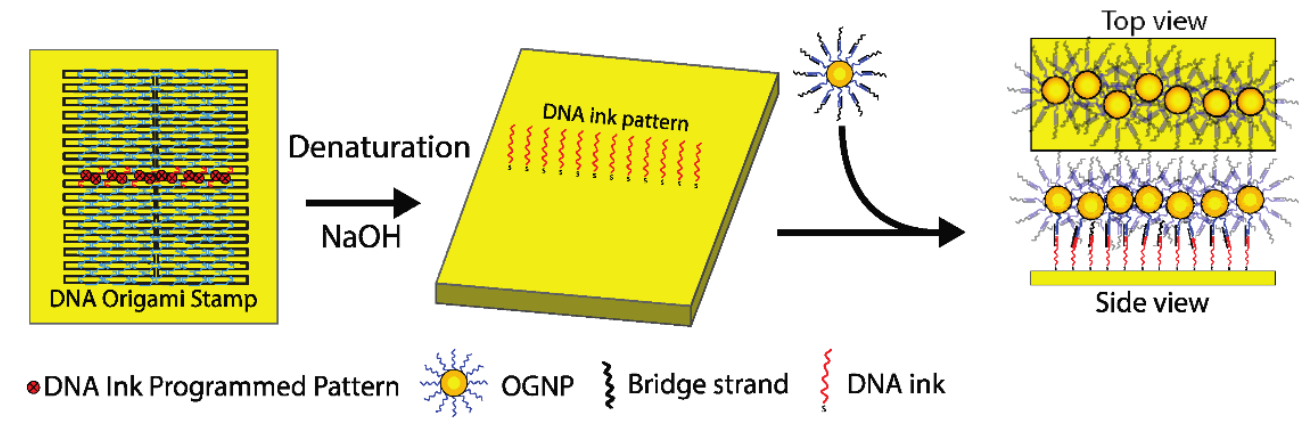

Figure 1. DNA-origami stamping process of a linear DNA ink programmed pattern on gold surfaces. The protocol describes the three basic steps of the stamping (1), unmasking (2), and development (3). (1) DNA-origami stamp is adsorbed on a gold surface. Then the DNA stamp is left over the surface until the thiol groups of the ink and anchor staples react with the gold surface; (2) The frame of the DNA stamp is denatured with $\mathrm{NaOH}$ and rinsed out to expose the DNA ink pattern. The DNA bridge was annealed directly to the OGNP; (3) Finally, the pattern is developed with the annealing of the OGNP-bridge sequence to the surface.

\section{Results and Discussion}

As proof-of-principle, we have created a linear pattern of thiol-modified staples on gold surfaces, a simple geometry that can be statistically analyzed in our experimental setup. Here, 12 staple strands were replaced by 5 -thiol-modified staples. Figure 1 shows the programmed positions for the ink staples within the DNA origami to print a line on the gold surface.

The formation of the linear pattern is revealed by the formation of bead-on-a-string-like structures after addition of gold nanoparticles conjugated with oligonucleotides (OGNP) that are complementary to the DNA ink pattern on the surface (Development step). OGNP-chain formation was determined by SEM imaging (Figure $2 a, b$ ). The DNA ink pattern was developed using OGNPs of $5 \mathrm{~nm}$ and $10 \mathrm{~nm}$ in diameter to investigate size-dependent effects on chain formation. The insets in Figure $2 b$ show selected chain images corresponding to each class of number of OGNP in a chain observed by SEM. Some of the chains do not contain straight OGNP alignments having zigzag-like shapes.

Statistical analysis of the distribution of nanoparticle alignment showed a decay of the frequency upon increment of number of OGNP in a chain and that the apparent, statistically significant maximum number of particles in a chain was significantly below to the 12 DNA ink programmed on the printed pattern [5]. The statistical analysis and Monte Carlo simulations were used to create a computational model. This model has provided insight into the key elements governing the stamping method, such as that the maximum apparent number of OGNP in a chain that a single DNA ink pattern can hold is due to geometrical factors affecting mainly the development step. These combined studies have also allowed to explain the zigzag behavior of some chain classes. Finally, our computational model was utilized to simulate formation of more complex patterns (Figure 2c,d) that could be used for future implementation of our method with tailored properties. Future studies could 
lead to the integration of this methodology within multiplexed microfluidic [6] and multipurpose read out systems [4], for the systematic analysis of biochemical assays.

a

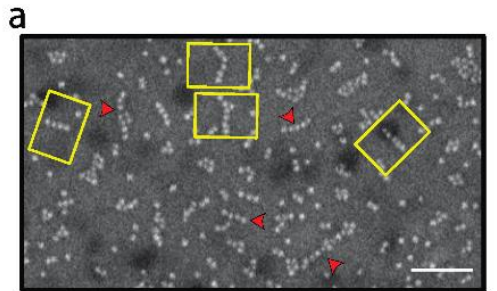

b

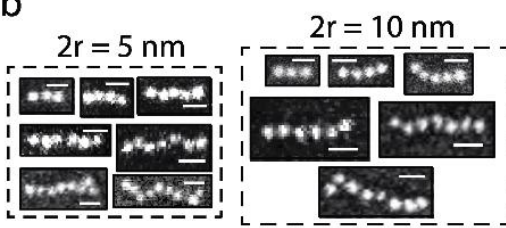

OGNP afixed to a DNA ink

Reduced hydrodynamic $\varnothing$

- OGNP-DNA ink hybridisation
C

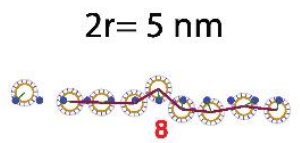

d

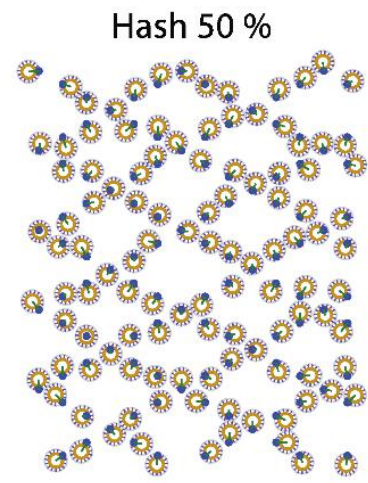

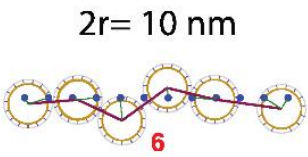

Space Invader

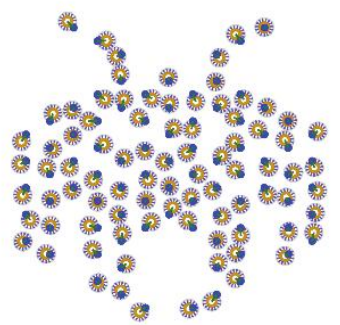

DNA ink well formed

Figure 2. Experimental data and Monte Carlo simulations after Development step. (a) SEM image of $10 \mathrm{~nm}$ OGNP aligned with the DNA-origami stamp method. The yellow rectangles depict the DNAorigami stamp frame domain $(100 \mathrm{~nm} \times 70 \mathrm{~nm})$. The red arrowheads point some OGNP chain alignment. Scale bar: $100 \mathrm{~nm}$. (b) SEM images corresponding to different chain classes (i.e., different numbers of OGNP aligned in a chain using 5 and $10 \mathrm{~nm}$ particles. $2 \mathrm{r}$ indicates the diameter of the gold nanoparticles. Scale bars: $20 \mathrm{~nm}$ (c) Selected results of CFS using 5 and $10 \mathrm{~nm}$ OGNP. The centres of the OGNP have been linked with a violet line to highlight the chain paths formed. The figures in red indicate the number of OGNP contained in the chain. (d) Monte Carlo PFS simulations of the development of the indicated DNA ink patterns using $4 \mathrm{~nm}$ (hash 50\%) and $3 \mathrm{~nm}$ (space invader) OGNPs.

\section{Conclusions}

We have demonstrated for the first time, that using DNA origami with chemically-modified staples in programmed positions, it is possible to print patterns with sub-10 $\mathrm{nm}$ precession on technologically relevant surfaces. Thereby, the DNA origami stamping method brings the opportunity for a more versatile and robust functionalization and patterning of surfaces for the creation of metamaterials [7] with applications in nanoelectronics and photonics, and the development of next generation biosensors.

Acknowledgments: We thank L. A. Bottomley for discussion. The Nanotechnology Platform at the IBEC for SEM technical support. This study was supported by the European Communities (FUNMOL, FP7-NMP-2133822), Spanish Ministry of Education (CTQ2010-20541) (IG, BM and RE), the Generalitat de Catalunya (2009/SGR/208 and 2009/SGR/505) (IG, BM and RE) and (2014 SGR 1442) (JS, MM), the CIBER-BBN (VI National R\&D\&I Plan 2008-2011) (IG, BM, MM, JS and RE), Iniciativa Ingenio 2010, Consolider Program, CIBER Actions, Instituto de Salud Carlos III with assistance from the European Regional Development Fund (IG, BM, RE, JS, MM), and from the European Research Council (FP/2007-2013)/ERC Grant Agreement n. 336917 (JDP). JDP acknowledges the support from the Serra Húnter Program.

Conflicts of Interest: The authors declare no conflict of interest.

\section{References}

1. Seeman, N.C. Nanomaterials Based on DNA. Annu. Rev. Biochem. 2010, 79, 65-87, doi:10.1146/annurevbiochem-060308-102244.

2. Rothemund, P.W. Folding DNA to create nanoscale shapes and patterns. Nature 2006, 440, $297-302$. 
3. Kershner, R.J.; Bozano, L.D.; Micheel, C.M.; Hung, A.M.; Fornof, A.R.; Cha, J.N.; Rettner, C.T.; Bersani, M.; Frommer, J.; Rothemund, P.W.K.; et al. Placement and orientation of individual DNA shapes on lithographically patterned surfaces. Nat. Nanotechnol. 2009, 4, 557-561, doi:10.1038/nnano.2009.220.

4. Scheible, M.B.; Pardatscher, G.; Kuzyk, A.; Simmel, F.C. Single Molecule Characterization of DNA Binding and Strand Displacement Reactions on Lithographic DNA Origami Microarrays. Nano Lett. 2014, 14, 16271633, doi:10.1021/nl500092j.

5. Gállego, I.; Manning, B.; Prades, J.D.; Mir, M.; Samitier, J.; Eritja, R. DNA-Origami-Driven Lithography for Patterning on Gold Surfaces with Sub-10 nm Resolution. Adv. Mater. 2017, 29, 1-7, doi:10.1002/adma.201603233.

6. Hsieh, K.; Ferguson, B.S.; Eisenstein, M.; Plaxco, K.W.; Soh, H.T. Integrated electrochemical microsystems for genetic detection of pathogens at the point of care. Acc. Chem. Res. 2015, 48, 911-920, doi:10.1021/ar500456w.

7. Kildishev, A.V.; Boltasseva, A.; Shalaev, V.M. Planar Photonics with Metasurfaces. Science 2013, 339, 1232009, doi:10.1126/science.1232009.

(C) 2017 by the authors. Licensee MDPI, Basel, Switzerland. This article is an open access article distributed under the terms and conditions of the Creative Commons Attribution (CC BY) license (http://creativecommons.org/licenses/by/4.0/). 